$18 \mid 2014$

NOVECENTO... E DINTORNI

Da Torino a Parigi: Laura Malvano storica e critica d'arte

\title{
Carlo Scarpa e Rob Mallet-Stevens: casa Sacerdoti e villa de Noailles
}

Carlo Scarpa et Rob Mallet-Stevens : " casa Sacerdoti » et la " villa Noailles » Carlo Scarpa and Rob Mallet-Stevens: "casa Sacerdoti" and "villa Noailles"

\section{Luciana Miotto}

\section{OpenEdition Journals}

Edizione digitale

URL: http://journals.openedition.org/cei/1926

DOI: 10.4000/cei.1926

ISSN: 2260-779X

\section{Editore}

UGA Éditions/Université Grenoble Alpes

\section{Edizione cartacea}

Data di pubblicazione: 30 mars 2014

Paginazione: 199-220

ISBN: 978-2-84310-268-4

ISSN: $1770-9571$

\section{Notizia bibliografica digitale}

Luciana Miotto, «Carlo Scarpa e Rob Mallet-Stevens: casa Sacerdoti e villa de Noailles», Cahiers d'études italiennes [Online], 18 | 2014, online dal 30 septembre 2015, consultato il 27 mars 2021. URL: http://journals.openedition.org/cei/1926 ; DOI: https://doi.org/10.4000/cei.1926 


\title{
CARLO SCARPA E ROB MALLET-STEVENS: CASA SACERDOTI E VILLA DE NOAILLES
}

\author{
Luciana Miotto
}

Su due disegni del progetto per la casa dell'avvocato Gino Sacerdoti (I9401943) a Venezia, Scarpa fa riferimento all'architetto francese, che tra il 1922 e il 1938 aveva realizzato in Francia una serie di opere.

In una grande tavola (026066) con vari disegni di sedie si notano in basso a destra tre schizzi relativi a casa Sacerdoti, tra cui una facciata e una pianta (fig. I) sotto la quale l'architetto scrive: «idea buona v Mallet Stevens». Sembra quasi che avesse cominciato a riflettere sulla soluzione da dare a quel progetto e ne avesse tracciato rapidamente i primi schizzi, sulla tavola su cui stava disegnando altro. E l'idea così materializzata gli fa venire in mente (o forse era già una sua ispirazione) una realizzazione di Mallet-Stevens: la villa a Hyères (fig. 2) nella costa Azzurra, costruita per il visconte Charles de Noailles nel 1924-1928. La casa è infatti riconoscibile, nello schizzo della facciata, per il gioco di volumi che si conclude con un'alta torre. In un secondo disegno (026062), una prospettiva della casa con la vista della stessa facciata (fig. 3), Scarpa ribadisce la fonte, scrivendo in basso a destra: «studiare meglio e togliere il carattere di plagio Mallet Stevens».

Le due piccole frasi, relative alla soluzione formale del progetto, sollevano degli interrogativi: perché Scarpa nel 1940 s'ispira a Mallet-Stevens? Perché proprio alla villa Noailles? Dove e come era venuto a conoscenza di quest'opera realizzata negli anni venti? Non esistevano pubblicazioni specifiche sulla villa, salvo un articolo del $1928^{1}$, in un numero di «Art et Décoration» che però Scarpa non possedeva. Tra i libri della sua biblioteca abbiamo comunque trovato la prima monografia su Mallet-Stevens

I. L. Deshaires, Une villa moderne à Hyères, in "Art et Décoration", juillet 1928, pp. I-8. 
scritta da Léon Moussinac ${ }^{2}$ e vari numeri di riviste degli anni trenta, dove erano pubblicate le altre sue opere. Scarpa conosceva quindi la produzione dell'architetto francese, in un certo senso lo ammirava $^{3}$ e forse trovava in lui delle affinità.

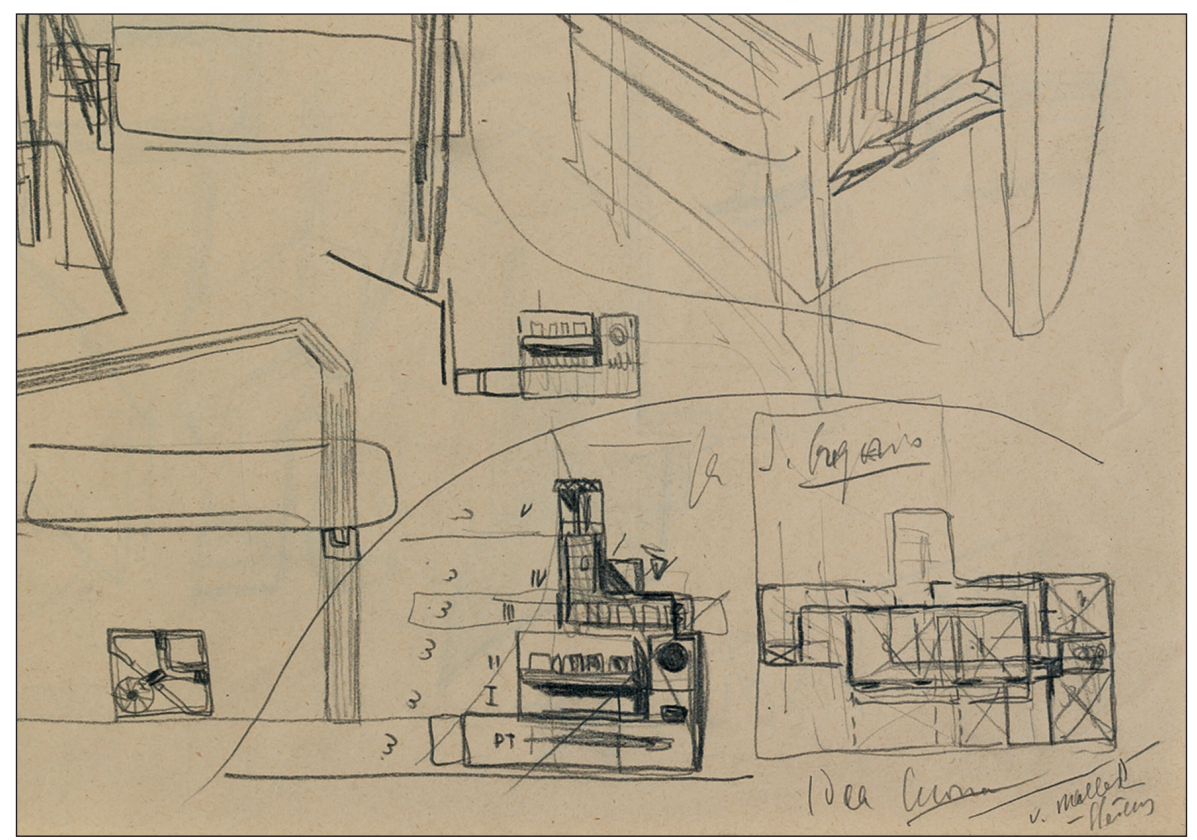

Fig. I. - C. Scarpa, Casa Sacerdoti (1940-1943), primi schizzi, dettaglio del disegno 026066.

Archivio Carlo Scarpa (infra ACS), proprietà del Museo Nazionale delle Arti del XXI secolo (infra MAXXI Archivi), conservato presso l'Archivio di Stato di Treviso, foto L. Baldin.

2. L. Moussinac, Mallet-Stevens, Paris, G. Crés, Les Artistes Nouveaux, I93I. Nella sua biblioteca c’è anche la piccola pubblicazione sulla villa Cavrois a Croix (nei pressi di Lilla), Une demeure 1934 architecte Rob Mallet-Stevens, éditions de l'Architecture d'Aujourd'hui, ma l'opuscolo gli fu donato da Philippe Duboy negli anni settanta.

3. Guido Pietropoli (a lungo suo collaboratore) ricorda che il «Professore» era soprattutto colpito dallo stile raffinato, dall'eleganza di Mallet-Stevens, e dal fatto che, a Parigi, aveva una via a suo nome con tutti gli edifici progettati da lui. 


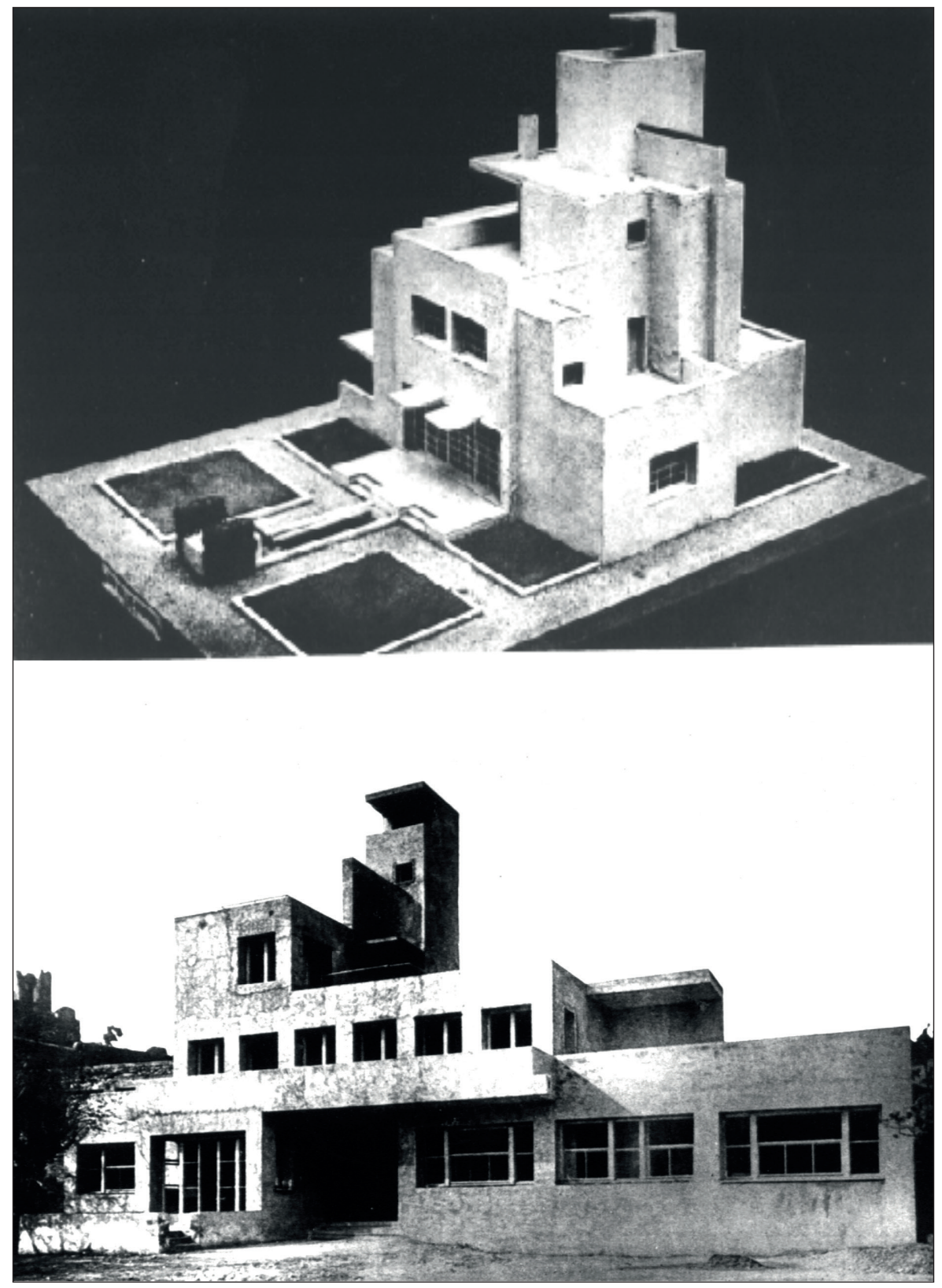

Fig. 2. - R. Mallet-Stevens, progetto "villa 1924", e villa Noailles a Hyères ancora in cantiere.

Da De Stijl et l'architecture en France, Bruxelles, Mardaga, 1985. 


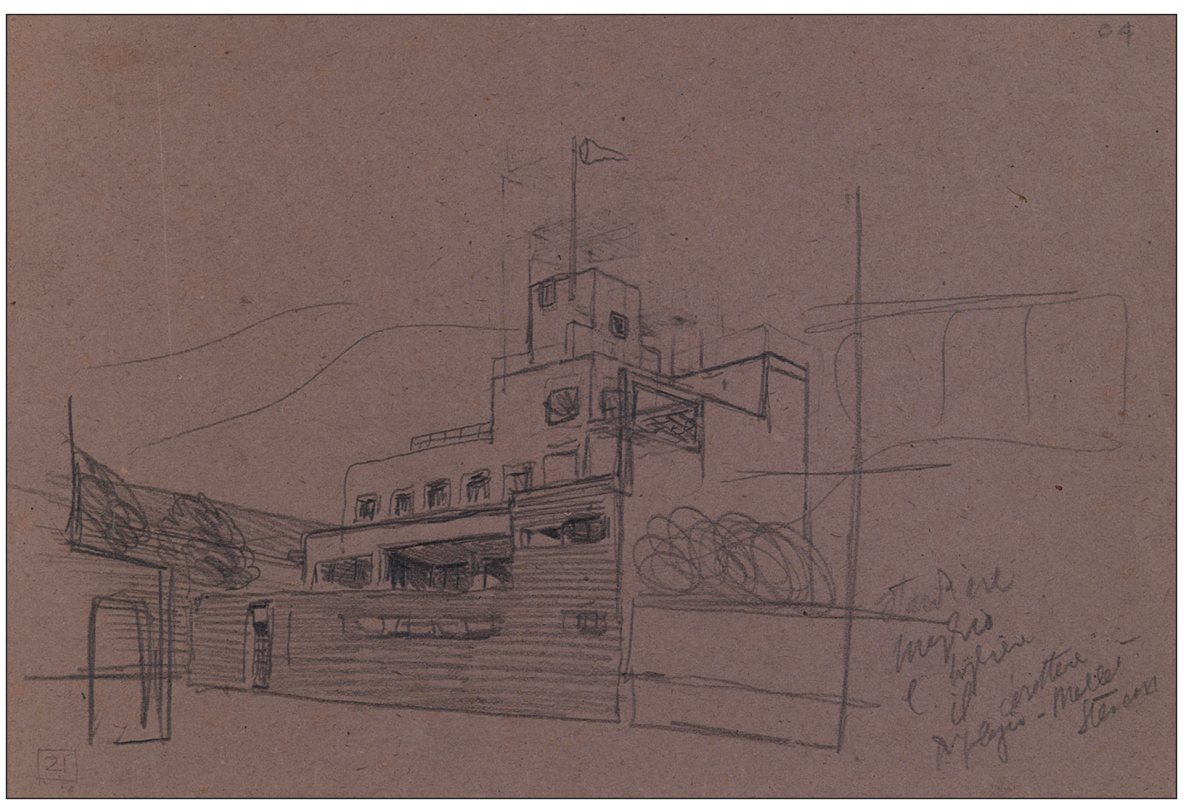

Fig. 3. - C. Scarpa, casa Sacerdoti, studio prospettico della facciata verso la calle, disegno 026062.

ACS, MAXXI Archivi, conservato presso l'Archivio di Stato di Treviso, foto L. Baldin.

\section{Le occasioni culturali di Mallet-Stevens tra il 1910 e il 1930 e di Scarpa tra il 1920 e il 1940}

I due architetti non sono certo affini per educazione e formazione, non solo perché Mallet-Stevens era cresciuto in un ambiente di maggiori interessi d'arte, culturali e commerciali ${ }^{4}$, ma anche per la differenza d'étà. Nato nel 1886 (vent'anni prima di Scarpa), aveva conosciuto durante gli studi di architettura e agli inizi della sua carriera l'ultima fase dell'Art Nouveau, tuttavia non furono gli esempi francesi di Guimard o dei seguaci della scuola di Nancy ad influenzarlo, bensì quelli della Secessione. Fondamentale per la sua formazione di architetto era stata la conoscenza diretta del

4. Il padre Maurice Mallet, esperto di pittura, fu uno dei primi a valorizzare gli impressionisti, la madre d'origine belga era figlia di Arthur Stevens, critico d'arte e collezionista, di cui due suoi fratelli erano pittori noti nell'ambiente artistico di Bruxelles. Sua figlia Suzanne Stevens, zia di Robert, aveva sposato il barone Adolf Stoclet, anche lui collezionista d'arte e committente di Hoffmann e di Klimt. Robert prese anche il nome della madre, Stevens, secondo la tradizione spagnolo-fiamminga. 
cantiere, e poi della realizzazione, del famoso palazzo Stoclet, costruito a Bruxelles tra il I905 e il I9II da Joseph Hoffmann.

Anche Scarpa era un grande ammiratore di Hoffmann, conosceva e ammirava il palazzo Stoclet, possedeva infatti le riviste su cui era stato pubblicatos, e lo aveva ampiamente illustrato nella famosa «Lezione sul liberty" del $1947^{6}$.

La villa Noailles e il progetto di casa Sacerdoti trovano la loro fonte proprio nella composizione volumetrica di quest'opera hoffmanniana e in quel concludersi con la torre. Per entrambi gli architetti l'interesse per Hoffmann si evolve, quale seguito logico, verso il cubismo, e poi verso De Stijl.

Per Mallet-Stevens quest'ultimo movimento fu determinante per la sua architettura, e per il modo di progettare. Curioso e interessato ai movimenti artistici contemporanei, egli ammirava logicamente anche Mackintosh et $W_{\text {right }}{ }^{7}$, e era al corrente dei lavori del gruppo De Stijl già prima di conoscerne il fondatore ${ }^{8}$.

Theo van Doesburg s'installa a Parigi nel maggio del 1923 per preparare la mostra Les Architectes du Groupe "De Stijl», alla galleria L'Effort Moderne del mercante d'arte Léonce Rosenberg, dove, dal is ottobre al I5 novembre, vengono esposti i progetti d'architettura più avanzati del movimento, realizzati da lui e da Cornelis van Eesteren. Ma a Parigi, come in Italia, imperava in quegli anni il «ritorno all'ordine» e quella stimolante mostra non ebbe successo.

Fu ad ogni modo in quell'occasione che Mallet-Stevens si legò d'amicizia con van Doesburg e ne propagandò le idee all'École Spéciale d'Architecture dove insegnava. In questa sede organizzò infatti la mostra L'Architecture et les arts qui s'y rattachent dal 22 marzo al 30 aprile 1924. Nella sezione architettura erano esposti i progetti di De Stijl già nella galleria L'Effort Moderne, i lavori degli allievi di Mallet-Stevens e una serie di progetti dei più celebri e impegnati architetti francesi che avevano accettato il suo invito a sostenere in tale modo la manifestazione. La mostra ebbe un buon successo; un cronista di «Art et Décoration» riportava: «Le principal attrait de l'exposition réside dans les travaux des élèves de Mallet-Stevens, ils n'admettent d'autre matériau que le ciment armé» 9 . Ma fu proprio

5. «L'Architecte, revue mensuelle de l'art architectural», I924; «Moderne Bauformen», agosto I929.

6. Cfr. «Les cahiers de la recherche architecturale», ${ }^{\circ}$ I9, I986, p. 38.

7. Su Wright scrive l'articolo Frank Lloyd Wright et l'architecture nouvelle, in "Wendingen», I925.

8. Si veda il saggio di B. Reichlin, Mallet-Stevens VS De Stijl, in Y.-A. Bois e B. Reichlin (a cura di), De Stijl et l'architecture en France, Bruxelles, Mardaga, 1985, pp. I09-I20.

9. Chroniques, in "Art et Décoration", avril 1924. 
a causa di questo insegnamento «moderno» che il docente venne licenziato. Alla mostra, i progetti degli allievi erano presentati con dei plastici, come ormai era in uso per il Salon d'Automne, e come l'aveva fatto molto efficacemente van Doesburg per la mostra da Rosenberg. Anche MalletStevens aveva esposto un modello di un progetto, la «villa I924" (fig. 2). Le Corbusier aveva reso conto della mostra nelle pagine de «L'Esprit Nouveau", e aveva spiegato le caratteristiche di quei progetti in questi termini:

Il s'agit exclusivement ici de constructions à réaliser en béton armé, on conçoit donc des formes procédant de la droite, de l'angle droit, de la verticale, de l'horizontale, art éminemment orthogonal. Le toit, bien entendu, n'existe plus [...]. Les façades s'ouvrent de baies largement distribuées. Les murs comptent pour leur surface seule, engendrant des volumes s'agençant les uns avec les autres, se pénétrant, se recoupant, s'alignant. Il n'y a plus de corniches, ni de moulures, il n'y a plus d'arcades ni de colonnes; le déblayage est fait avec le plus fol enjouement d'un âge sans pitié ${ }^{10}$.

Mallet-Stevens aveva fatto partecipi i suoi studenti della lezione di De Stijl e delle teorie di Theo van Doesburg ${ }^{\text {II }}$, li aveva spinti a sperimentare nei loro modelli la rottura della scatola chiusa, a liberare le tre dimensioni tradizionali, a lavorare sulle superfici nello spazio tenendo conto della quarta dimensione: il tempo. Anche il progetto della sua «villa 1924" era un'esplorazione formale per raggiungere una «coesione plastica» e una «nuova armonia».

In realtà era un doppio esercizio: sul tema della villa e sull'applicazione delle ricerche di De Stijl. Esercizi su cui lavorava concretamente, dato che stava mettendo a punto il progetto definitivo della villa a Hyères, di cui avrebbe iniziato il cantiere nell'aprile di quell'anno. Nel dicembre esce un suo articolo in cui teorizza il contenuto dei due progetti:

L'architecture est un art essentiellement géométrique. Le cube est la base de l'architecture [...]. Une maison, un palais sont composés d'un ensemble de cubes. [...]. De nos jours, le béton armé transforme complètement les problèmes qu'a à résoudre le constructeur. Mille formes sont permises, des silhouettes imprévues surgissent, étranges parfois mais rationnelles, sincères. [...]. L'architecte moderne peut faire autre chose qu'un bloc compact fait de pierre, de bois, de fer, de zinc, de fonte, de marbre, de stuc; il peut "jouer" avec une succession de cubes monolithiques. [...]. Les saillies, les décrochements rectilignes formeront de grands plans d'ombres et de lumière. [...]. Surfaces unies, arêtes vives, courbes nettes, matières polies, angles droits; clarté, ordre. C'est la maison logique et géométrique de demain ${ }^{12}$.

IO. Le Corbusier, L'exposition Spéciale d'Architecture, in «L'Esprit Nouveau», n 23, 1924.

II. Cfr. B. Zevi, Poetica dell'architettura neoplastica, Il liguaggio della scomposizione quadridimensionale, Torino, Einaudi, 1974 (1953).

I2. R. Mallet-Stevens, testo senza titolo, in «Le Bulletin de la vie artistique», nº 23, déc. 1924, pp. 532-534. 
La «villa I924» è infatti un organismo plastico, composto da una successione di cubi che avvolgono, sul davanti e ai lati, la torre-belvedere. Simile è la composizione della villa Noailles, come pure l'esterno del «laboratorio dell'ingegnere», progettato da Mallet-Stevens nel 1923 per la scenografia del film di Marcel L'Herbier L'Inhumaine, in cui una serie di volumi si conclude con un'alta torre, e l'entrata è decorata in una perfetta composizione De Stijl.

Negli anni tra il 1920 e il I926, Scarpa studia alla Reale Accademia delle Belle Arti di Venezia ${ }^{13}$. Mentre la cultura italiana di quel periodo soffriva delle limitazioni imposte dal regime fascista, l'ambiente veneziano offriva qualche stimolante sollecitazione, soprattutto con le manifestazioni delle Biennali e il passaggio d'importanti artisti e critici, come Virgilio Guidi, Arturo Martini, Gino Severini, o Lionello Venturi, che Scarpa ha modo di conoscere e con alcuni di stringere amicizia. Con il pittore Mario Deluigi, compagno e amico fraterno dell'Accademia, frequenta il Circolo artistico ai Piombi, dove si discutevano gli avvenimenti artistici, mentre alla libreria Ravenna, al Campanile, si potevano consultare libri e riviste vietati dal regime. In un'intervista, Deluigi ricorda:

Il fascismo negava ogni apporto culturale straniero. Per esempio quello che si faceva a Parigi, città allora all'avanguardia delle ricerche pittoriche, io lo venivo a sapere attraverso le riviste "Verve» e «Minotaure» che l'architetto Carlo Scarpa, mio compagno di stanza, mi faceva leggere. Fu così che nel 1926 andai a Parigi [...]. Ho appreso così de visu quello che era il movimento cubista ${ }^{\mathrm{I4}}$.

Scarpa doveva conoscere il movimento De Stijl e le manifestazioni parigine attraverso le pubblicazioni che possedeva o che vedeva dal libraio Ravenna, come il numero di «L'Architecture Vivante» del 1925 interamente consacrato a quel gruppo. Di certo, come l'amico Deluigi, in quegli anni si appassiona per il cubismo e per Le Corbusier, come infatti confessa:

Abbiamo dovuto fare una certa fatica per liberarci dalla nostra formazione scolastica [...]. Per me [...] é stata una fortuna trovare il volume intitolato Vers une architecture di Le Corbusier al termine della scuola [1926]: rappresentò un'apertura dell'anima; da allora le condizioni spirituali maturarono totalmente ${ }^{\mathrm{Is}}$.

Le discussioni con Deluigi e i resoconti dei suoi viaggi a Parigi (nel I926 e 1937) gli apportavano altre conoscenze. Dalla visita dell'esposizione

13. Sulla formazione di Scarpa di quel periodo, si veda C. Sonego, Carlo Scarpa: gli anni '20, in K. W. Forster e P. Marini (a cura di), Studi su Carlo Scarpa 2000-2002, Venezia, Marsilio, 2004, pp. 27-83.

I4. G. Bianchi, L'attività didattica di Mario Deluigi. Imparare a vedere l'arte, Venezia, Cavallino, 20Io, p. 7.

15. C. Scarpa, Può l'architettura essere poesia? Testo della conferenza a Vienna nel 1976, in Carlo Scarpa. Opera completa, a cura di F. Dal Co e G. Mazzariol, Milano, Electa, 1984, p. 283. 
universale del 1937 e della contemporanea mostra I maestri dell'arte indipendente al Petit Palais, Deluigi — come spiega Giovanni Bianchi — riporta la conferma del valore degli «elementi plastici del cubismo». Il vero tramite di questi interessi è per entrambi il rapporto d'amicizia con Gino Severini, e Deluigi lo riconoscerà in una lettera del I94I a lui diretta: «[...] me l'hai insegnato tu, ne parli sempre tu nei discorsi e negli scritti precedenti che l'arte è un fatto plastico e null'altro $[\ldots]{ }^{16}$. Anche Scarpa ne era convinto, lo ribadirà in alcune affermazioni future, come l'ammirazione per un "fatto architettonico e plastico», la convinzione che "per ottenere qualcosa bisogna inventare dei rapporti e [...] immaginare il cromatismo delle cose...» ${ }^{17}$, o ancora la constatazione del risultato di «effetti plastici ${ }^{18}$ su alcune sue opere.

Negli anni tra il I926 e 1940, tra la fine dell'Accademia e il progetto di casa Sacerdoti, lo troviamo impegnato in varie attività: insegna, progetta e disegna vetri. Queste esperienze saranno fondamentali sia per la sua formazione culturale che di architetto, in particolare il lavoro di collaboratore presso i vetrai di Venezia - prima ai Maestri Vetrai Muranesi Cappellin \& C. (1927-I932), poi alla Società Venini (1933-1942). Oltre a disegnare vetri, impararne le tecniche e l'uso dei materiali, per tali ditte allestisce anche negozi e mostre ${ }^{19}$, dapprima alla Mostra Internazionale delle Arti Decorative di Monza del 1927 e del I930, poi alla Triennale di Milano del I936 e del 1940, importanti occasioni di aggiornamento sulle tendenze architettoniche contemporanee, italiane e straniere. Alle mostre di Monza esponevano i razionalisti italiani e gli architetti del Werkbund tedesco e austriaco; in quella del 1930, la sezione dell'Austria era stata curata da Hoffmann, che forse Scarpa incontra allora ${ }^{20}$. Come dirà nell'intervista del 1976, Hoffmann era stato l'artista che "più aveva ammirato e più lo aveva istruito».

Benché non avesse realizzato alcun allestimento alla V Triennale del 1933 è plausibile pensare che l'avesse visitata dato l'interesse dei temi esposti ${ }^{21}$. Le varie sezioni comprendevano: la mostra di arte decorativa, l'esposizione di architettura, la pittura murale che orna gli ambienti monumentali (a

I6. In G. Bianchi, L'attività didattica, cit., p. 8.

17. C. Scarpa, Arredare, in Carlo Scarpa. Opera completa..., cit., p. 282.

18. ID., Volevo ritagliare l'azzurro del cielo, in Carlo Scarpa. Frammenti 1926-1978, "Rassegna», n 7, I98I, p. 82 .

19. Cfr. O. Lanzarini, Note sull'esperienza di Carlo Scarpa alla Triennale di Milano 1927-I960, in K. W. Forster e P. Marini (a cura di), Studi su Carlo Scarpa, cit., pp. 87-I38.

20. Ibid., pp. 89-9I.

2I. VTriennale di Milano. Programma, Milano, S.A.A.E., I933; Catalogo ufficiale della V Triennale di Milano, a cura di Agnoldomenico Pica, Milano, Ceschina, I933.

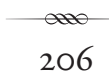


cura di Mario Sironi), la mostra dell'abitazione moderna (a cura di Giò Ponti) con le opere dei razionalisti italiani, e l'architettura internazionale (presenti dieci paesi stranieri). In quest'ultima sezione erano esposte opere dei più importanti architetti moderni, anche in mostre monografiche, tra cui Wright, presentato in Italia per la prima volta, Dudok, Hoffmann, Loos, Gropius, Mendelshon, Mies van der Rohe, Lurçat, Le Corbusier con Pierre Jeanneret. Nella Galleria delle Nazioni erano esposte anche alcune opere di Mallet-Stevens, ma non la villa a Hyères: nel pannello dedicato alla Francia due edifici della rue Mallet-Stevens, la sua casastudio e quella degli scultori Martel entrambe del 1927; nella sezione «opere tipiche» la sede dell'Alfa Romeo a Parigi (1925), il Casinò di SaintJean-de-Luz (I928), la distilleria a Istanbul (I93I); nella sezione «ville e giardini», la villa Cavrois a Croix (I93I-I932) 22 .

Se nel 1924 Mallet-Stevens si era pubblicamente impegnato a difendere l'architettura moderna organizzando la famosa mostra all'Ecole Spéciale, Scarpa nel I93I scrive insieme ai razionalisti veneziani la lettera a sostegno del Movimento italiano per l'architettura razionale, i cui orientamenti erano stati ufficialmente criticati da Marcello Piacentini, in particolare l'uso del cemento armato, proprio come i detrattori dei progetti degli allievi di Mallet-Stevens. Nel passo della lettera relativo alla difesa del materiale, si riconosce il pensiero di Scarpa, specie nel concetto di architetto moderno, che palesa una certa affinità con De Stijl:

Nell'epoca presente tutti usano il nuovo materiale da costruzione; il quale indica meglio di qualunque programma o teoria estetica, la via sulla quale bisogna battere il passo. Sarà grande artista quegli che, adoperando il cemento armato e domandolo alle esigenze e agli scopi razionali dell'edificio, saprà introdurre l'elemento spirituale e fantastico, creandone allora l'espressione artistica ${ }^{23}$.

Negli anni trenta Scarpa arricchisce la sua biblioteca in particolare di libri e di riviste di architettura francesi - come "L'Architecture d'aujourd'hui», "Art et Décoration» — e soprattutto tedesche, come «Moderne Bauformen», «Deutsche Kunst und Dekoration». C’è comunque da notare che tra i suoi libri, oltre a testi sulla pittura, prevalgono quelli di letteratura e di poesia specialmente in francese.

I progetti di cui si occupa allora riguardano arredamenti di case e negozi, spesso studiati con Mario Deluigi. Una loro realizzazione, la casa

22. Ringrazio Alessandra Castelbarco per le informazioni sulle Triennali e per la ricerca sulle relative foto, reperite presso l'archivio della Triennale.

23. Adesioni al movimento razionalista, in "Il Lavoro Fascista», I9 maggio I93I. 
dell'antiquario Ferruccio Asta, è pubblicata in «La Casa Bella» da Edoardo Persico ${ }^{24}$ che definisce i due progettisti «ottimi decoratori italiani».

A Venezia, la conferenza di Le Corbusier nel 1934, a Palazzo ducale, su «La leçon de la gondole» fu un avvenimento importante, forse Scarpa vi assistette. L'influsso di Le Corbusier si nota comunque nella sua prima realizzazione di architettura, il restauro e sistemazione di Ca' Foscari (I9361937), vi partecipa anche Deluigi con l'opera La scuola, un affresco «del tutto innovativo e ispirato a soluzioni formali cubiste» ${ }^{25}$. L'evoluzione artistica dei due amici procedeva di pari passo.

Se Mallet-Stevens si interessava al cinema, firmando delle scenografie che la critica chiamava "futuriste» ${ }^{26}$, i veneziani, a scapito del loro provincialismo, non solo avevano le Biennali ma dal 1932 anche l'Esposizione internazionale d'Arte Cinematografica, nata nell'ambito della XVIII Biennale, e diventata dal ' 35 una manifestazione annuale. Sappiamo che Scarpa amava il cinema, e non ci stupirebbe che avesse visto L'Inhumaine, dato che L'Herbier era un po' di casa alla «mostra», nel '36 aveva fatto parte della prima giuria internazionale.

Nel 1940 Scarpa inizia il progetto di casa Sacerdoti; nell'aprile si apre a Milano la VII Triennale, l'ultima sotto il fascismo, dove sono esposti i complessi dell'E42 e le nuove città di fondazione, ma solo per un brevissimo tempo, in giugno la manifestazione viene sospesa: l'Italia è entrata in guerra come alleata della Germania, aggredendo proprio la Francia. È forse per esprimere solidarietà con questo paese, di cui amava svisceratamente la cultura, che Scarpa "plagia» un'opera di un architetto francese? Ma perché proprio la villa a Hyères?

\section{La casa de Noailles a Hyères (progetto 1923 , realizzazione 1924-1928)}

In vista del matrimonio con Marie-Laure, il visconte Charles de Noailles decide di costruire una casa nel Sud della Francia per viverci d'inverno ${ }^{27}$. Amante di opere d'arte d'avanguardia, e amico di artisti contemporanei, desidera una casa «moderna». Nel 1922 è alla ricerca di un architetto capace

\footnotetext{
24. Arredamento a Venezia di De Luigi e Scarpa, in "La Casa Bella», n 55, 1934, pp. 34-37.

25. G. Bianchi, L'attività didattica, cit., p. 5 .

26. «Futurismo - Un dramma passionale dell'anno 1950» è il titolo italiano del film L'Inhumaine.

27. In un'intervista a Grasse (il I7 agosto 1979) dove il visconte risiedeva, mi raccontava che il desiderio di vivere una parte dell'anno nel Sud della Francia, all'aria aperta, praticando anche vari sport, gli era nato dalla preoccupazione che sua moglie si ammalasse di tubercolosi come il suocero. Il terreno, situato su una collina dominante la cittadina d'Hyères e il mare — già sito dell'antico convento dei Bernardini, detto «Clos de Saint Bernard» —, era un dono della madre.
} 
di rispondere a tale aspettativa, ma allora ben poche erano le realizzazioni moderne in Francia. Vorrebbe affidarne il progetto a Mies van der Rohe, che incontra in Germania, ma Mies ha troppi impegni. Va anche a Dessau, passa una giornata al Bauhaus incontrando professori e allievi, ma non sa decidersi. Un altro tentativo lo fa a Parigi con Le Corbusier, ma il carattere «molto dominatore» dell'architetto svizzero lo scoraggia. Ai primi del 1923, in occasione di una visita al Museo delle Arti Decorative, chiede consiglio al direttore Louis Metman, vecchio amico di famiglia, che gli segnala Mallet-Stevens: "ho visto solo un giovane che faccia cose serie qui, è l'architetto che ha esposto questo "padiglione d'aereo-club" al Salon d'Automne» ${ }^{28}$. De Noailles gli affida il progetto, Mallet-Stevens lo inizia nella primavera del 1923 e avvia il cantiere nel I924. Ma durante i lavori, de Noailles si rende conto che la casa è troppo piccola, chiede l'aggiunta di quattro camere che verranno situate in un'ala a ovest del nucleo iniziale, collegata a questo con un corridoio retrostante. Nel 1926 una seconda ala, con davanti un giardino pensile, viene aggiunta egualmente a ovest ma molto più in alto, e collegata all'ala sottostante con un corridoio a rampa. Sul retro del corpo iniziale viene inoltre aggiunto un vasto ambiente (l'atelier di de Noailles), illuminato dall'alto. La casa è inaugurata nel gennaio dello stesso anno, ma continuerà ad ingrandirsi con altre aggiunte, come la piscina coperta e la sala da ginnastica nel 1928. Giunta a questo stadio, la villa è diventata un vero «castello» e, proprio come tale, Man Ray la filma nel famoso cortometraggio Les Mystères du Château de Dé.

Hyères è la prima opera di Mallet-Stevens realizzata, che immaginiamo sofferta date le numerose modifiche decise dal proprietario anche senza la sua approvazione. Ciò spiegherebbe le relative poche pubblicazioni dedicate alla villa, e la poca pubblicità voluta forse dall'architetto stesso ${ }^{29}$. Il complicato rapporto col committente s'instaura fin da principio; in reazione a una prima proposta dell'architetto, in una lettera del 5 luglio 1923 , de Noailles gli ribadisce le intenzioni iniziali:

28. Ne aveva esposto il plastico al Salon d'Automne del I922, e poi nella hall del museo dove lo vide de Noailles. Il padiglione, un primo tentativo di scomposizione volumetrica, con incertezze negli incastri ma con una buona resa delle nude susperfici, doveva comunque aver colpito il visconte per il suo aspetto di modernità.

29. Mostre e articoli sulle sue opere sono soprattutto dedicati agli edifici della rue Mallet-Stevens (I927) e alla villa Cavrois (I93I-I932); l'interesse per l'architettura di Mallet-Stevens si ferma con gli omaggi in onore della morte (1945). Segue un lungo oblio rotto più di dieci anni dopo dal breve articolo di G. Habasque, Deux précurseurs français : Chareau et Mallet-Stevens, in "L'EIL", $\mathrm{n}^{\circ} 60$, déc. 1959 , in cui alla villa è fatto appena un accenno. Solo dagli anni settanta inizia un vero interesse per la sua opera, con la tesi di C. Bonnefor, Rob Mallet-Stevens. Positions et contradictions de l'architecture internationale, thèse de $3^{\mathrm{e}}$ cycle, Paris, I974. 
J'ai envie de bâtir une maison extrêmement moderne, mais par moderne j'entends employer tous les moyens modernes pour arriver au maximum de rendement et de commodité. Je ne compte pas plus sacrifier un pouce de fenêtre pour obtenir une façade Louis XVI que pour obtenir une façade moderne intéressante ${ }^{30}$.

Nell'immediata risposta (il 6 luglio I923), Mallet-Stevens lo tranquillizza: "Croyez bien que rien ne sera sacrifié en plan pour arriver à une façade architecturale, si la disposition intérieure après étude est bien, les façades logiquement seront bien».

Siamo nell'estate del 1923, l'architetto riprende da capo il progetto della villa, nell'autunno vede la mostra di De Stijl alla galleria L'Effort Moderne e incontra Theo van Doesburg. Già affascinato dai princìpi di quel movimento, aveva appena sperimentato la straordinaria ricchezza "plastica» del gioco dei volumi e dei piani nelle scenografie esterne del film L'Inhumaine. Ne ritroviamo l'applicazione anche nella villa: se la pianta non é ancora «libera», la distribuzione delle funzioni segue i dati del programma sposando quelli fisici del sito; i volumi che ne risultano si integrano così al terreno e al paesaggio, e gli spazi interni ed esterni si compenetrano.

Come insegnava van Doesburg, l'aspetto "plastico" dell'architettura é ora ottenuto "dalla quarta dimensione dello spazio-tempo" " ${ }^{3 \mathrm{I}}$ : la villa è infatti tutta orchestrata sui percorsi, è su questi che si agganciano le varie parti, creando così un'architettura «informe», libera, non più costretta da una forma prestabilita ${ }^{32}$. Ciò permetterà a de Noailles di realizzare aggiunte sopra aggiunte, alla casa di diventare un «castello», alla sua architettura «dinamica» di trasformarlo in labirinto, e a Man Ray di girare sui suoi percorsi, interni ed esterni, il famoso film, di cui ricorda la genesi in questi termini:

Aux vues d'une seule photo de la villa, mon esprit se mit à travailler malgré moi. Les formes cubiques du château me firent penser au titre d'un poème de Mallarmé: Un coup de dés jamais n'abolira le hasard. Ce serait le thème du film et son titre aussi : Les Mystères du Château de Dés3.

Mentre de Noailles si preoccupava delle comodità della casa e che il suo aspetto fosse sì «moderno» ma non ostentatorio, Mallet-Stevens tentava di realizzare la sua visione dell'architettura ${ }^{34}$.

30. Lettera di Charles de Noailles a Rob Mallet-Stevens del 5 luglio 1923. La corrispondenza relativa al progetto e ai problemi della villa è stata da me consultata e annotata presso l'archivio di M. Edmond de la Haye Jousselin, nipote del visconte, subito dopo l'incontro di Grasse del 1979.

3I. Th. van Doesburg, L'évolution de l'architecture moderne en Hollande, in «L'Architecture Vivante», automne-hiver I925, p. I8.

32. Ivi, p. I6.

33. Man Ray, Autoportrait, Paris, Laffont, 1964, p. 248.

34. Si veda il suo scritto in "Le Bulletin de la vie artistique», art. cit. 
Come nell'esempio del plastico della «villa I924", anche a Hyères «l'insieme dei cubi» trova la sua conclusione nella torre. Questa tuttavia diventerà un elemento di discordia tra l'architetto e il committente. Fin dal progetto, de Noailles era stato scettico sulla sua funzionalità, ma, supponiamo, soprattutto sul suo aspetto ostentatorio. La fa comunque costruire (gennaio 1925), la prova, e poi decide di sopprimerla ${ }^{35}$ : «depuis que je suis monté je me rends bien compte que je n'y monterai plus jamais et que son côté architectural m'ennuiera plus de jour en jour», ma Mallet-Stevens lo supplica di non toccarla: «J'ai fait des croquis pour m'imaginer la maison sans la tour et l'on obtient alors un ensemble sans relief, sans silhouette et sans expression... $»^{36}$. Ma l'idea di modernità del visconte è molto più radicale:

Je comprends parfaitement tout ce que vous me dites pour cette maudite tour qui nous divise et à qui je reconnais aussi bien que vous de grandes qualités du point de vue de l'apparence de la maison. Mais si vous vous en souvenez je vous ai dit dès le premier jour que je ne pourrai jamais supporter quoi que ce soit dans la maison ayant un but seulement architectural et que je cherchais une maison infiniment pratique et simple où chaque chose serait combinée du point de vue de l'utilité. Vous voyez pourquoi maintenant, chaque fois que je regarde la tour qui donne forcément à la maison un côté architectural, elle représente tout ce que je désirais éviter ${ }^{37}$.

Al di là dei giusti meriti del risultato d'insieme della villa, quest'affaire della torre mette in luce il lato formalista dell'architettura di MalletStevens, che tuttavia non gli impediva di realizzare dei «pezzi» assolutamente esemplari come l'atelier, l'aggiunta del 1926. Giustamente Cécile Briolle lo definisce «l'espace intérieur le plus caractéristique de la modernité dans la villa Noailles» ${ }^{38}$. Un ambiente di alta qualità plastica, che non solo testimonia la comprensione della lezione di De Stijl da parte di Mallet-Stevens, ma anche la sua conoscenza di Wright: l'atelier ricorda infatti l'Unity Temple.

Scarpa realizzerà un ambiente di altrettanta qualità plastica in omaggio a Mondrian e a De Stijl, il posto telefonico della Telve a Venezia nel 1950.

Un'altra affinità tra i due architetti era la loro ammirazione del Giappone, entrambi l'avevano scoperto e studiato sui libri. Mallet-Stevens ammirava sopattutto l'assenza di decorazioni e le pareti nude delle case

35. Nella fig. 2, foto del cantiere 1924, la torre è intera, nel 1925 verrà soppressa la parte del belvedere.

36. Lettera di Rob Mallet-Stevens a Charles de Noailles del 23 aprile I925.

37. Lettera di Charles de Noailles a Rob Mallet-Stevens del 29 aprile 1925.

38. C. Briolle, La villa Noailles, in C. Briolle, A. Fuzibet e G. Monnier, La villa Noailles. Rob MalletStevens, Marseille, Parenthèses, 1999 (I990), p. 7I. 
giapponesi ${ }^{39}$, a cui si era ispirato nella villa. Aveva inoltre riposto i quadri della collezione del visconte in un ambiente apposito ${ }^{40}$ per essere esposti - come i «kakemono» - solo quando si volevano ammirare. Eccezionalmente farà decorare le pareti della «stanza dei fiori» dall'amico van Doesburg, ma quell'insieme costituiva un'opera d'arte in sé, chi voleva vederla doveva andare in quell'ambiente appositamente.

All'esposizione L'Architecture et les arts qui s'y rattachent del 1924 van Doesburg aveva presentato, in forma di volantino, il «V Manifesto di De Stijl» intitolato Vers une construction collective. Anche negli scritti precedenti aveva affermato i "princìpi collettivi del gruppo», gli artisti dei diversi campi - pittura, scultura, architettura - dovevano lavorare insieme per mettere a punto un nuovo concetto d'arte. Mallet-Stevens, per la realizzazione della villa a Hyères, opera in modo analogo. Riunisce infatti vari artisti, che collaborano, ciascuno nel proprio campo, al risultato finale. Alcuni sono invitati dallo stesso de Noailles, come Guévrékian per la realizzazione del giardino triangolare "cubista», la maggior parte da Mallet-Stevens, in particolare Louis Barillet per i vetri, Jo Bourgeois et Francis Jourdan per gli arredi di varie camere e sale, Pierre Chareau per la "camera all'aperto» e Theo van Doesburg per la decorazione pittorica di cui abbiamo detto.

Ovviamente anche il committente, a parte certe decisioni «da proprietario", collaborava all'équipe dando l'avallo alle scelte degli artisti proposti da Mallet-Stevens, lasciandolo provare materiali e meccanismi nuovi nel suo primo cantiere e accettando di sperimentare l'architettura moderna nella propria casa. Se Mallet-Stevens inventava dei meccanismi inediti, come i serramenti vetrati della piscina che «sorgevano dal suolo provenendo dall'interno della struttura di cemento" per ripararla nei giorni di vento e di freddo, de Noailles, sul muro di chiusura del giardino di fronte all'entrata, suggeriva d'inserire delle aperture per inquadrare il paesaggio. Nell'intervista del $1979^{41}$ diceva di aver visto Pienza. Mallet-Stevens le disegnerà in sintonia con lo stile «cubista» della villa.

39. Cfr. Y. Brunhammer, Robert Mallet-Stevens architecte d'intérieur, in B. Buyssens et al. (a cura di), Rob Mallet-Stevens architecte, Bruxelles, Archives d'Architecture moderne, 1980, pp. 99-IIo.

40. Man Ray li aveva filmati (dal retro), appesi su dei pannelli grigliati come si usa oggi nei depositi museali. 4I. Vedi supra, n. 27. 


\section{Casa Sacerdoti (progetto 1940-1943)}

Nel catalogo delle opere pubbblicato in Carlo Scarpa. Opera completa, troviamo ben tre sistemazioni di case a Venezia per l'avvocato Gino Sacerdoti. Dal riordino e studio dei disegni condotti da Orietta Lanzarini ${ }^{42}$ risulta che la prima sistemazione, realizzata nel I937, riguarda l'arredo e la trasformazione in atelier di pittore (altra attività del Sacerdoti) di un pièd-à-terre a San Gregorio, non lontano dalla Salute, verso le Zattere. La seconda del I940-I94I, egualmente realizzata, concerne il riordino del I e II piano del palazzo di proprietà del committente, situato a Santa Maria del Giglio nei pressi della Fenice.

La terza sistemazione, quella che qui ci interessa, è rimasta invece allo stadio di progetto; si tratta del restauro e della trasformazione di una casa ad uso appartamenti, contornata da un giardinetto, situata egualmente a San Gregorio, in calle Monastero. Dai disegni non si capisce lo stato preesistente della casa, ma su due disegni si legge una prima versione del progetto, in cui Scarpa organizza gli appartamenti su quattro piani, in due corpi adiacenti affacciati sul giardino. Un maggior numero di disegni (32) concerne invece una seconda soluzione, in cui la casa viene ampliata e completamente riorganizzata. È in questa seconda versione che Scarpa s'ispira a Mallet-Stevens.

Dai disegni e da alcune note manoscritte si deduce che il programma consisteva precisamente nella sistemazione di un piano per la «rappresentanza», un altro per un uso abitativo più privato, e un terzo per un appartamento più piccolo per gli ospiti. In ogni piano si dovevano prevedere $\mathrm{i}$ relativi servizi. Anche il pianterreno era destinato a servizi, probabilmente a depositi, come di norma nelle case veneziane. Il progetto non va oltre la fase di studio in cui le distribuzioni d'uso richieste non sono ancora del tutto decise; nelle piante infatti si leggono le varie opportunità, mentre nelle prospettive, grandi e piccole, Scarpa studia l'impatto dei possibili volumi.

I primi piccoli schizzi (due piante e un alzato), disegnati sul bordo in basso della grande tavola, disegno 026066 (fig. I) — già accennati precedentemente - sembrano essere la genesi del progetto con già l'idea del riferimento alla villa Noailles, come si legge nella scritta: idea buona $v$ Mallet-Stevens. Lidea, per la quale Scarpa associa l'architetto francese allo

42. Che qui ringrazio vivamente per avermi fornito tutte le informazioni relative a questi tre progetti, in parte pubblicati nell'opuscolo della mostra Carlo Scarpa. Lo spazio dell'abitare. Disegni scelti I93I-I963, da lei curata per il Centro Carlo Scarpa, Archivio di Stato di Treviso, nel 2008. 
schizzo della facciata, è sicuramente la torre e i volumi che vi si aggregano sotto e di lato, come nella villa a Hyères. Anche la pianta di questa presenta delle similitudini con quella di casa Sacerdoti, così nel corpo scale posto in entrambe sul retro e in un volume esterno. Simile è pure la posizione della piccola scala che conduce al belvedere sopra la torre; nel progetto di Scarpa essa serve inoltre a collegare il terzo piano. La villa Noailles comporta invece un piano di meno.

L'indecisione della distribuzione e localizzazione degli appartamenti si legge in parte nel disegno 026063 (fig. 4), che presenta lo studio del II piano insieme a una parte del III, come specifica una nota manoscritta di Scarpa: «qui schizzo del II e del III». In un'altra scritta, in alto del foglio a destra, egli elenca la destinazione degli ambienti nei vari piani secondo «due» varianti. L'indecisione riguarda la zona notte dell'abitazione padronale che potrebbe situarsi o al primo piano o al secondo, o solo la sua camera al terzo. ${ }^{43}$ Oltre che per le note manoscritte, questo disegno è interessante anche per i piccoli schizzi prospettici in alto a sinistra, e sotto. I due in alto mostrano la volumetria della casa al di sopra delle due ali di muro che circondano il terreno, nei quali Scarpa sembra voler verificare il rapporto dei nuovi volumi col muro di cinta, specie con l'ala che fiancheggia la calle, su cui si apre il portone d'accesso alla proprietà.

Lo schizzo prospettico, in basso a destra, mostra invece un dettaglio della facciata del I piano, con abbozzata la sistemazione dell'entrata. Scarpa la collega direttamente al livello della calle, tramite una scala che prende l'avvio subito a destra del portone d'ingresso alla proprietà. Come si vede anche nel disegno (37656) della pianta del I piano sistemato per la «rappresentanza», la rampa si addossa al muro della calle e raggiunge un vasto pianerottolo che continua nel varco (o portico) dell'entrata a tale appartamento.

Altre due prospettive (studiate in due disegni separati), mostrano la casa vista dalla calle, da due angolature opposte. Il disegno 02606I (fig. 5), dove si nota l'indicazione della rampa che porta al I piano, presenta una prima composizione dei volumi della facciata principale, come la struttura aggettante della terrazza del II piano (sulla sinistra), il corpo a destra che s'innalza dal muro della calle e termina in una sorta di altana, i volumi del III piano e la torre. Il disegno evidenzia lo stadio del progetto in cui si è scelto di situare il volume più importante del III piano (appartamento degli ospiti o camera del padrone?) alla sinistra della torre-belvedere.

43. Queste possibilità di spostamenti d'uso sono indicate anche nella tavola 37655 in due serie di note manoscritte, poste sopra e sotto la pianta del II piano qui disegnata. 
È nella seconda prospettiva, disegno 026062 (fig. 3), che la composizione della casa appare più vicina alla villa di Mallet-Stevens (con la torre accorciata). Forse è l'ultimo disegno del progetto, qui il volume più importante del III piano è ora situato a destra della torre, il volume aggettante della terrazza del II piano è prolungato lungo tutta la facciata, e il corpo a destra termina a gradoni, contribuendo a concatenare meglio i volumi intorno alla torre e dare all'insieme una visione più dinamica. Non a caso proprio in questo disegno Scarpa scrive il secondo riferimento all'architetto francese: «Studiare meglio e togliere il carattere di plagio MalletStevens». Torniamo quindi al quesito iniziale: perché, in questo progetto dei primi anni quaranta, egli s's ispira alla villa Noailles di Mallet-Stevens?

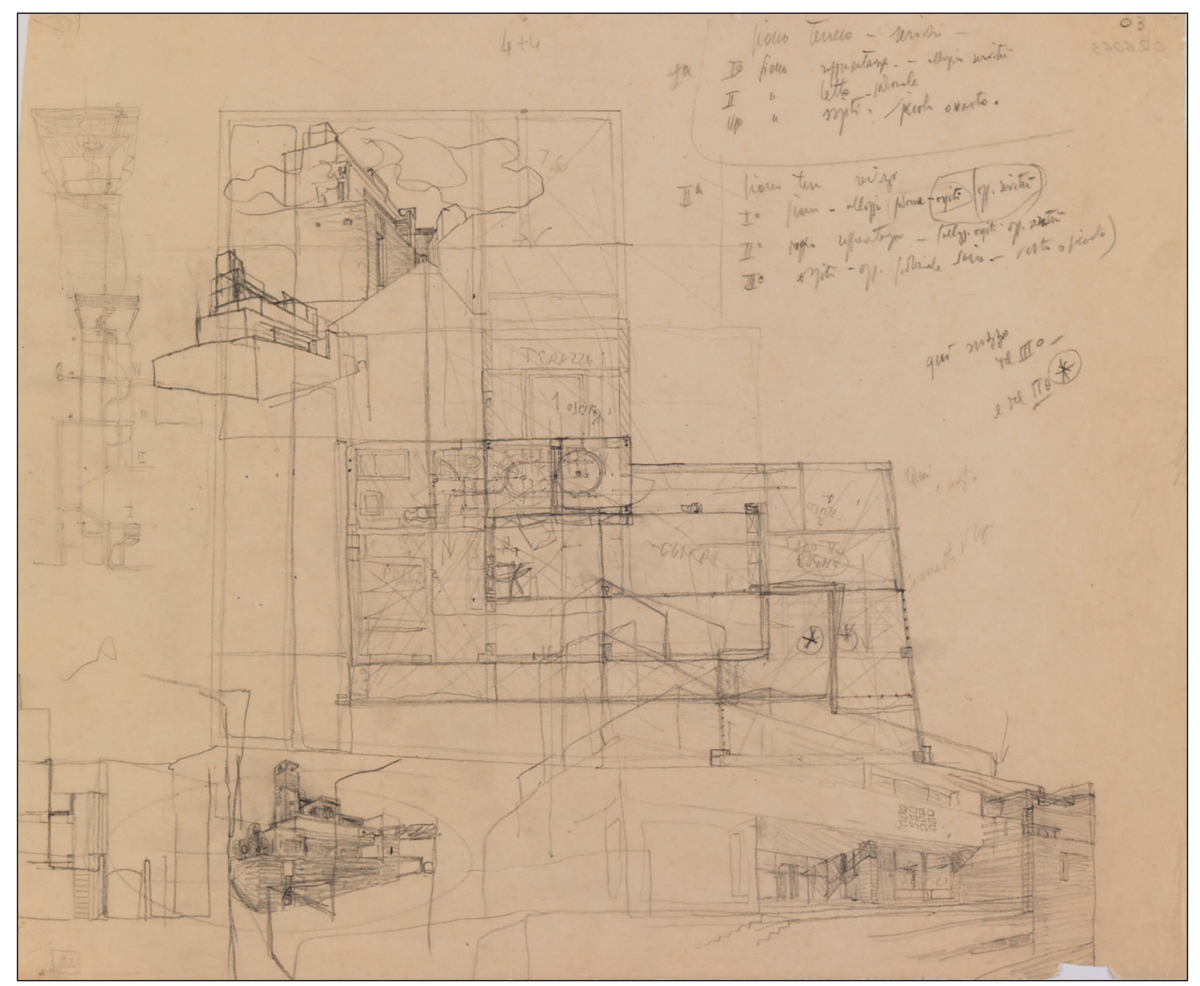

Fig. 4. - C. Scarpa, casa Sacerdoti, pianta del II e III piano con note manoscritte e studi prospettici, disegno 026063 .

ACS, MAXXI Archivi, conservato presso l'Archivio di Stato di Treviso, foto L. Baldin. 


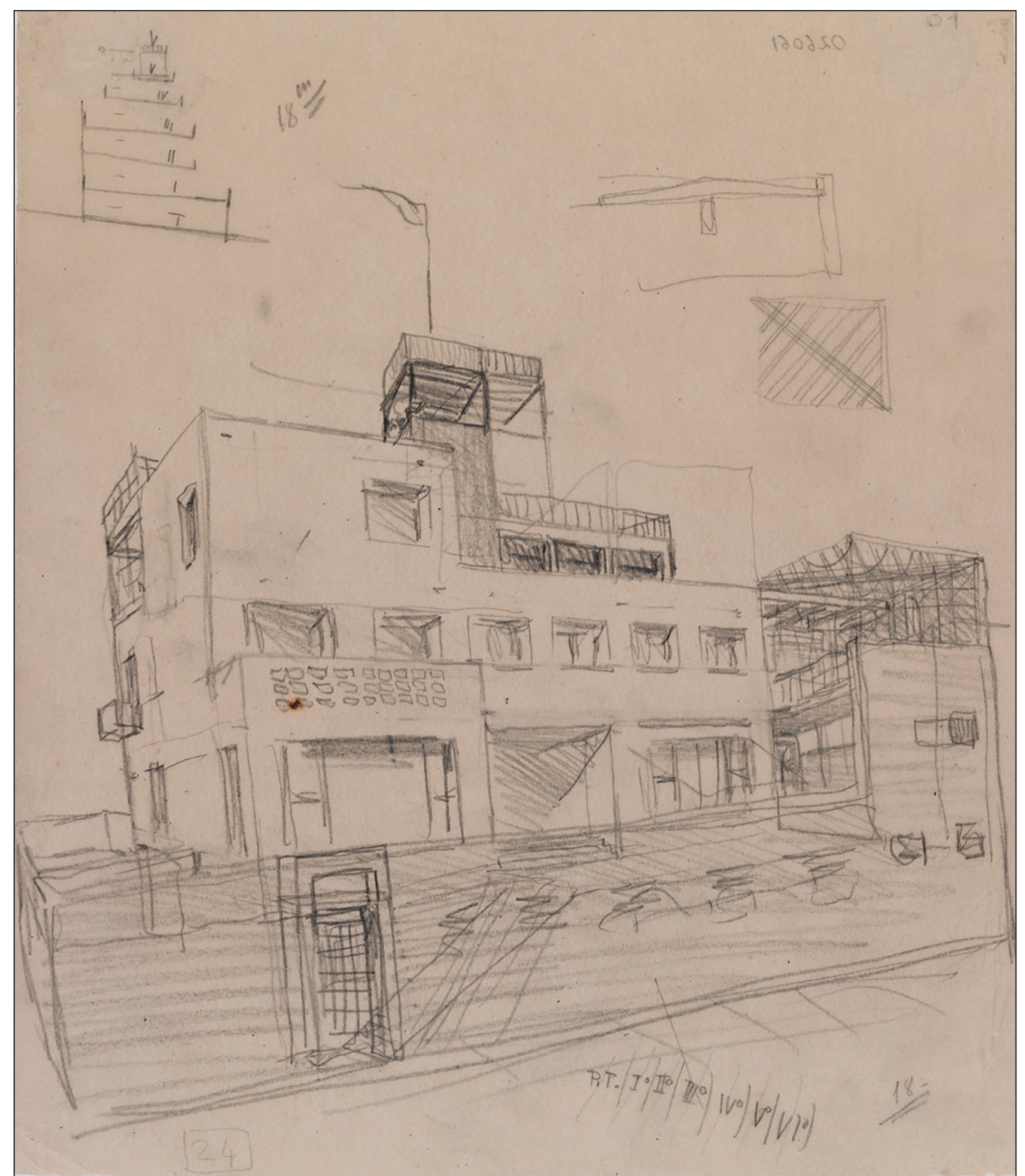

Fig. 5. - C. Scarpa, casa Sacerdoti, studio prospettico della facciata verso la calle, disegno 02606I.

ACS, MAXXI Archivi, conservato presso l'Archivio di Stato di Treviso, foto L. Baldin.

\section{Il film di Man Ray, la causa del «plagio»?}

Né il tema, né il programma, né il sito dei due progetti presentano condizioni analoghe. Mentre l'architettura della villa francese nasce da precise condizioni ambientali e programmatiche, a cui l'architetto risponde con 
un linguaggio espressivo derivato dall'evoluzione della sua cultura, il progetto della casa veneziana pare non appigliarsi a nessuna considerazione del genere, non è una dimora per vivere in modo nuovo, all'aria aperta e con possibilità di praticare vari sport, né per passare gli inverni, ma una casa signorile per viverci tutti i giorni, e a Venezia. L'unico elemento che abbia una funzione comune è la torre col belvedere, decisa in entrambe le case per vedere il mare, anche se poi de Noailles il belvedere, troppo alto, lo elimina.

Come scrive Orietta Lanzarini, «nella biografia di Scarpa sono documentabili numerosi episodi nei quali il contatto con una fonte provoca un riscontro» ${ }^{44}$. Guido Pietropoli spiega inoltre che "durante ogni innamoramento Scarpa subiva un processo di identificazione totale che lo portava ad appropriarsi non tanto delle semplici forme esteriori o dei compiacimenti estetici del suo occasionale maestro, bensì del carattere specifico di quell'artista o architetto» ${ }^{45}$. La forte analogia di casa Sacerdoti potrebbe essere un riscontro immediato con una fonte, provocato da un innamoramento forse doppio o conseguente, cioé sia della villa che dell'architetto. Ma quale "clic» aveva provocato tale innamoramento?

È difficile pensare che Scarpa abbia avuto una conoscenza approfondita della villa attraverso mostre o riviste di architettura, poiché, come abbiamo accennato, era stata poco pubblicata. Anche del suo architetto, negli anni ' 40 non si parlava quasi più; ormai ammalato si era ritirato dalla professione e, a causa della guerra, aveva anche abbandonato Parigi e la sua casa-studio nella mitica rue. Insomma, tra il 1938 e il I940, pochi ormai si ricordavano di Mallet-Stevens. Ci siamo allora chiesti se il coup de foudre per la villa e per il suo architetto, invece che dalla cultura architettonica, non gli fosse piuttosto venuto dal cinema, provocato proprio dalla visione del film di Man Ray, Les Mystères du Château de Dé.

Non abbiamo insistito abbastanza sul ruolo culturale del cinema all'epoca. Tra gli architetti, Mallet Stevens era stato uno dei primi ad intuirlo. A Parigi, nel 192I, era stato fondato il «Club des Amis du Septième Art» ${ }^{46}$ con lo scopo di affermare che il cinema era un'arte, anzi, la sintesi di tutte le arti, e per divulgarne la conoscenza; tra i suoi membri troviamo Mallet-Stevens, Fernand Léger, Maurice Ravel, Jean Cocteau, e altri artisti già affermati nei rispettivi campi. Grazie alle loro azioni, anche il cinema

44. O. Lanzarini, Note sull'esperienza di Carlo Scarpa, cit., p. 95.

45. G. Pietropoli, Il disegno nell'opera di Carlo Scarpa, in G. Beltramini, K. W. Forster e P. Marini (a cura di), Carlo Scarpa. Mostre e Musei 1944-1976. Case e Paesaggi 1972-1978, Milano, Electa, 2000, p. 59. 46. Cfr. M. Louis, Mallet-Stevens et le cinema I9I9-I929, in B. Buyssens et al. (a cura di), Rob MalletStevens architecte, cit., p. I26. 
entra al Salon d'Automne dello stesso anno, dove molti film sono presentati e commentati dallo stesso Mallet-Stevens. Nel dicembre del 1922 i membri fondano la rivista "Gazette des Sept Arts», diretta dallo scrittore e giornalista Ricciotto Canudo, il più attivo del gruppo e il più esperto di cinema. Mallet-Stevens è tra i redattori, scrive vari articoli e disegna la grafica del giornale. La sua esperienza di «decoratore di film» (una decina tra il 1919 e il 1929) lo convince del ruolo che il cinema poteva avere per la conoscenza e la propagazione delle arti, in particolare per l'architettura moderna. Nel 1928 pubblica Le Décor moderne au Cinéma, dove difende l'idea che "le cinéma doit être un art moderne» ${ }^{4}$.

Non sappiamo se il «Club des Sept arts» avesse stimolato la fondazione di associazioni simili anche in Italia, come avvenne in altre città europee, comunque a Venezia nel 1932, con la nascita della prima Esposizione internazionale di Arte Cinematografica, il cinema è considerato «un'arte», e nel 1938 viene organizzata la prima grande retrospettiva, dedicata proprio al "Cinema francese dal ı89i al 1933". Ma il film di Man Ray non era nell'elenco dei film presentati. Scarpa non poté quindi vederlo in quell'occasione. È comunque fuori dubbio che anche il cinema aveva una certa influenza nell'entourage degli artisti veneziani frequentati da Scarpa e da Deluigi. Arturo Martini, ad esempio, se ne era ispirato per la famosa scultura Donna che nuota sott'acqua (194I-1942) ${ }^{48}$.

Pensiamo quindi che anche Scarpa si sia ispirato alla villa Noailles influenzato dal film di Man Ray, visto con molta probabilità a Milano nel 1940, nell'ambito della VII Triennale, per la quale il nostro architetto aveva allestito un ultimo stand della Venini ${ }^{49}$. In questa Triennale era stata presentata anche una grande «Mostra del Cinema», la prima nell'ambito di una manifestazione di architettura e di arti decorative, organizzata da

47. R. Mallet-Stevens, Le décor moderne au cinéma, Paris, Massin \& Cie, 1928, p. 6.

48. Scarpa e Deluigi erano legati ad Arturo Martini da un forte rapporto d'amicizia; invitato ad insegnare all'Accademia, lo scultore trevigiano aveva incaricato Scarpa nel I94I di sistemargli la sua futura casa alle Zattere. Nel 1942 i due amici veneziani allestiscono la sua mostra alla XXIII Biennale, in una sala del Palazzo Centrale (cfr. O. Lanzarini, Carlo Scarpa. L'architetto e le arti, Venezia, Marsilio, 2003, p. 23), scegliendo anche le sculture da esporre, tra cui la Donna che nuota sott'acqua (1942), l'opera più sconvolgente, realizzata proprio allora da Martini. Essa rappresentava «un'espressione fisiologica del nudo»; le forme fisiologiche — così definite da Deluigi - erano per Martini quelle che potevano «immaginare una forma sotto acqua, che spostamento che succede [sic! in veneziano], tanto per specchio d'acqua quanto per movimenti insoliti" (citato in G. Bianchi, L'attività didattica, cit., p. 23). L'idea di questa scultura gli era venuta da una sequenza del film di W. Van Dyke, White Shadows of the South Seas (1928), cfr. A. Martini, Colloqui sulla scultura I944-I945, raccolti da Gino Scarpa, a cura di Nico Stringa, Treviso, Canova, 1997. Sono grata a Carlo Montanaro di avermi segnalato questo aneddoto, che conferma l'interesse che avevano per il cinema i nostri artisti; lo ringrazio anche per le informazioni relative alla Fondazione Mario Ferrari.

49. Si veda O. Lanzarini, Note sull'esperienza di Carlo Scarpa, cit., p. 96. 
due giovani architetti, appassionati cinefili e animatori della Fondazione Mario Ferrari di Milano, Luigi Comencini e Alberto Lattuada, con la collaborazione di Henry Langlois, il fondatore della Cinémathèque Française. Con tale manifestazione era stato ulteriormente riconosciuto al cinema il suo posto fra le arti. Se tuttavia, nella lista dei materiali presentati e tra le foto di alcuni fotogrammi dei film visionati, non si è trovato traccia del film di Man Ray (trattandosi forse di un cortometraggio), sappiamo comunque che negli archivi della Fondazione Mario Ferrari ce n'era una copia, molto probabilmente lasciata allora da Langlois ${ }^{50}$.

Un importante indizio del presunto interesse per questo film da parte di Scarpa è anche la sua passione per Mallarmé. Come ricorda Kurt W. Forster egli era un appassionato lettore dei suoi poemi; continuerà per tutta la vita a raccoglierne le «edizioni più autorevoli»" ${ }^{5}$. Col poema $U n$ coup de Dès jamais n'abolira le Hasard, del I897, il poeta simbolista aveva rivoluzionato la sintassi e la grafica tradizionali ben prima dei futuristi. Nelle pagine da lui composte, aveva fatto scorrere le parole sul percorso dei dadi, scomponendole e ricomponendole sullo spazio bianco, creando "una bellezza enigmatica» ${ }^{2}$.

Nel film Les Mystères du Château de Dé, Man Ray fa la stessa cosa con l'architettura «cubista» della villa e il gioco dei «dadi» ispirato a Mallarmé, ogni colpo di questi svela un «mistero» del «Castello». Come scrive Michel Louis:

L'architecture de Mallet-Stevens, filmée sous un angle inattendu, sous une lumière nouvelle, crée sur l'écran des formes abstraites, comme autant de traces littéraires s'adonnant aux joies de la poésie. La caméra fixe un objet, une surface, un volume, jusqu'à ce que l'attention grandisse, jusqu'à ce qu'ils prennent un aspect mystérieux ${ }^{53}$.

Salvo in un'inquadratura presa da lontano, l'architettura della villa non é mai filmata integralmente ma solo per parti e dettagli, che evidenziano con maggiore forza il suo linguaggio plastico. Secondo Luc Wouters, Man Ray «mette in luce un aspetto dell'opera di Mallet-Stevens spesso trascurata dai critici: la sua dimensione poetica» ${ }^{54}$. Può l'architettura essere

50. La Fondazione Mario Ferrari dal 1947 diventa "Cineteca italiana».

5I. K. W. Forster, Alla ricerca dell'architectus absconditus, in K. W. Forster e P. Marini (a cura di), Studi su Carlo Scarpa, cit., p. I5. Si veda anche, nello stesso volume, Ch. VoGel, "Élever enfin une page à la puissance du ciel étoilé». Scarpa-Mallarmé : l'architetto poeta alla luce del poeta architetto (pp. I83-196).

52. Nel museo di Castelvecchio di Verona, Scarpa aveva composto la texture marmorea del Sacello, decidendone i colori con i dadi.

53. M. Louis, Mallet-Stevens et le cinéma, cit., p. 138.

54. L. Wouters, Cinéma et architecture, in J. F. Pinchon (a cura di), Rob Mallet-Stevens. Architecture, Mobilier, Décoration, Paris, Action Artistique de Paris-Ph. Sers, 1986, p. II3. 
poesia? Scarpa l'aveva forse intravista nella villa di Mallet-Stevens attraverso le inquadrature di Man Ray, e se n'era ispirato nel primo progetto di casa che gli era stato commissionato. Ma una volta disegnato, si accorge di aver imitato l'architetto francese troppo "formalmente», e di quel "plagio» si mette in guardia lui stesso. 